\title{
The roles of CPG phase modulation and reflexive muscular patterns in balance recovery during walking - a simulation study
}

\author{
Yu Ikemoto* and Wenwei Yu \\ Department of Medical System Engineering, Chiba University, Chiba, Japan
}

(Received 6 March 2008; final version received 22 May 2008)

\begin{abstract}
Most walking assist systems reported are not available for real world environments where frequent perturbations are caused by slips, uneven terrain, slopes and obstacles. It is evident that humans are able to cope with such perturbations with reflexes that cause unconscious, relatively fixed muscular response patterns to perturbations within a short period of time. In our previous study, we showed that artificial reflexes could improve the perturbation resistance for simulated walkers, though the roles of different reflexive mechanisms were not quantitatively clarified. In this study, we focused on the different roles of reflexive muscle responses and the $\mathrm{CPG}$ phase modulation mechanism. By proposing and evaluating two stability criteria through a series of simulation experiments, we revealed different roles for two mechanisms in the simulated walkers. These will not only further increase the possibility of realising artificial reflexes for paralysed individuals, but also bring new insights into the field of motor control.
\end{abstract}

Keywords: reflexes during walking; walking simulation model; walking assist; functional electrical stimulation

\section{Introduction}

Recently, walking assist systems, such as robotic systems (Kawamoto et al. 2003) and functional electrical stimulation (FES) for hemiplegic walking (Bajd et al. 1997; Tong and Granat 1998; Yu et al. 2002) have been widely studied for the purpose of improving daily living for paralysed individuals. However, most systems were unable to address the perturbations resulting from uneven terrain, slips, slopes and obstacles, which frequently occur in daily-life walking; as they have not taken these perturbations into consideration, they are not yet suitable for practical use in real-life situations.

However, it is evident that humans can cope with such perturbations, especially when they cannot be predicted or perceived in advance, by means of reflexes (Zehr and Stein 1999) which cause relatively fixed, unconscious muscular response patterns to perturbations within a short period of time ranging from several tens of milliseconds to $200 \mathrm{~ms}$.

Our ultimate goal is to realise artificial reflexes in realworld walking support systems for paralysed individuals, whose afferent and efferent neural pathways are usually weakened so that the reflexive system is also impaired to a certain degree. This goal requires both a qualitative and quantitative understanding of human reflexive mechanisms during walking.
Reflexes of different functional organs and limbs (e.g. upper limbs (Cathers et al. 2004), hearts (Nakamura and Saito 1992) and lower limbs (Zehr and Stein 1999)) in different contexts (e.g. during flexion/extension (Cathers et al. 2004), during free fall (Bisdorff et al. 1999) and during walking (Zehr and Stein 1999)) have been studied in the fields of kinesiology and neuroscience. It has also been shown that flexor reflexes play an important role in locomotion, and these reflexes were implemented in several commercially available FES systems (Quintern 2000).

Although the reflexive responses to perturbation during walking have been an object of study for quite some time (Berger et al. 1984; Dietz et al. 1987; Zehr and Stein 1999), most studies were concerned with muscle activity recording and data analysis, through which several working hypotheses were generated. For example, electroneurograms of high-spinal-curarised cats were recorded and analysed to show that the stimulation of flexor reflex afferents could induce a clear resetting of the locomotion rhythm (Schomburg et al. 1998). However, it is almost impossible to test this hypothesis using the same methods in humans.

Thus, the spatiotemporal relation among neuro-control mechanisms, muscle activities and physical motions remains unknown. Moreover, there is no widely accepted theory on the underlying neural mechanisms of the reflexes

\footnotetext{
*Corresponding author. Email: yikemoto@graduate.chiba-u.jp
} 
during walking, nor are there clear experimental results that could be directly referenced in the disciplines of physiology and motor control. However, neuro-control-level understanding and verification are necessary to artificially realise reflexive responses to perturbation during walking.

Our basic hypothesis is that if the muscle activity profile of the reflexive responses to perturbation during walking can be acquired via non-invasive measurement, and if a neuromusculoskeletal walking simulation model able to present conformable behaviour to human normal walking could be developed, albeit without the reflexive mechanism pre-wired (as they are still unknown), parts of the candidates for the underlying neural mechanisms can be clarified by investigating which candidate can match the measured muscle activity profile.

In our previous study (Yu and Ikemoto 2007), we investigated reflexive responses during walking through the following methods:

(1) Acquiring muscle activity profiles during normal walking and slip-perturbed walking by recording and processing electromyographic (EMG) signals of several walking-related muscles in a human gait experiment.

(2) Developing a central pattern generator (CPG)based neuromusculoskeletal simulation model. Computer simulation has been employed as an approach to study the role of afferent information during human (Taga 1994) and animal walking (Prochazka and Yakovenko 2001). In an animal study, virtual reflexes were realised by a set of if-then rules, and the gait of cat walking with and without the virtual reflexes were compared. The results showed that walking with virtual reflexes was more stable and perturbation resistive. However, there are few studies employing a hybrid approach coupling human walking simulation with human gait experiment data.

(3) Comparing joint trajectories of the simulation model with those of a human subject during normal walking to verify the simulation model's conformity with human walking.

(4) Using muscle activity profiles of reflexive responses (defined as muscular reflexive patterns in the present study) extracted from EMG data recorded for slip-perturbed walking in the human gait experiment to construct a rapid responding pathway.

The results indicated that the simulation model could display behaviour resembling that of normal human walking, and, on the occurrence of a slip perturbation, together with the CPG phase modulation, the rapid muscular response could improve perturbation resistance and maintain balance for the simulated walker.
Although these results were quite encouraging, the roles of different reflexive mechanisms have not yet been quantitatively clarified. However, understanding the roles of these functional mechanisms is important not only from the viewpoint of assistive engineering, but also for possible scientific insights into the field of motor control.

In the present study, we focused on the different roles of the reflexive muscle responses and the CPG phase modulation mechanism. By using the human walking simulator developed in our previous study, a series of simulation experiments were performed to investigate the roles in perturbation resistance played by two functional mechanisms, i.e. muscular reflexive patterns and $\mathrm{CPG}$ phase modulation strategies, as well as the afferent feedback pre-wired in human walking models. Qualitative evaluation was performed to compare the different functional mechanisms.

For the quantitative evaluation, we used two stability criteria. One is the energy stability margin (Messuri and Klein 1985), which is used to evaluate static postures. However, human walking and balance recovery are apparently dynamic processes, and a static stability criterion may not correctly reflect the essence of these functions. Thus, we proposed energy difference, calculated from the rotational energy of lower limbs, to evaluate the dynamic aspect of stability.

The remainder of this article is organised as follows. Firstly, the muscle activity profiles of reflexive responses (defined herein as muscular reflexive patterns) extracted from EMG data of slip-perturbed walking in previous human gait experiments (Cathers et al. 2004; Yu and Ikemoto 2007) as well as the structure of the walking simulation model are described. Two simulated walkers, corresponding to different reflexive mechanisms, are also described. Next, the two stability criteria are defined. In Section 3, several experimental results comparing the two walkers' capability to cope with perturbations are examined. Finally, the interplay between the three functional mechanisms is discussed.

\section{Materials and methods}

\subsection{Muscular reflexive responses}

The muscle activity profiles of reflexive responses can be extracted from EMG data recorded from slip-perturbed walking in previous human gait experiments (Cathers et al. 2004; Yu and Ikemoto 2007).

The difference between muscular activities during normal walking and perturbed walking was compared to a threshold to determine the onset of reflex responses. The threshold was defined using the mean and standard deviation (SD) of the first $2.5 \mathrm{~s}$ of data from the subtracted activity profile, i.e. the point at which the amplitude goes beyond mean $\pm 3 \mathrm{SD}$ was considered the onset time. Additionally, for EMG recordings, the data after $2.54 \mathrm{~s}$ would be scanned for the onset determination. The 0.04-s gap was set 
Table 1. Latencies of the selected muscles.

\begin{tabular}{lccccc}
\hline Muscles side & Gluteus medius & Vastus lateralis & Semi tendinosus & Anterior tibial & Gastroc nemius \\
\hline Slip side (ms) & 149 & 175 & 178 & & 176 \\
Another side (ms) & 143 & & 88 & 116 & 176 \\
\hline
\end{tabular}

Note: '/' indicates the side on which the muscles do not satisfy the conditions.

according to the shortest muscle latency possible for reflex responses (Nakamura and Saito 1992).

The latency data was further processed to extract muscular reflexive responses. It is noted that effective latency could not be detected for all muscles and all subjects. Thus, only the muscles for which an effective latency could be detected for more than 5 of 10 trials and more than 7 of 10 subjects were selected as the ones that should be activated during reflexive responses (Table 1). The selected muscles and their averaged latencies were designated the muscular reflexive patterns.In similar studies (Berger et al. 1984; Dietz et al. 1987), the split-belt treadmill was employed to study the corrective reactions to unpredictable one-sided deceleration and acceleration perturbations during walking. The EMG signals of two muscles, the tibialis anterior (TA) and gastrocnemius, were recorded and analysed, and our results showed the same temporal activation sequence on the contralateral side for both. That is, TA was activated first, followed by gastrocnemius. TA was activated at a latency of $65 \mathrm{~ms}$; however, our TA latency was $116 \mathrm{~ms}$. This may have been due to differences in deceleration time, i.e. the treadmill could realise deceleration within $60 \mathrm{~ms}$, whereas the deceleration time of the split-belt walking machine was $100 \mathrm{~ms}$.

\subsection{Simulation models}

Two simulation models were developed. One was named the Normal Walker (Figure 1), which receives a command from the central nervous system and consists of a CPG model, a musculoskeletal model and a sensory feedback module. The other was designated the Reflexive Walker (Figure 2), having the same basic elements of the Normal Walker, but with an additional reflex mechanism modulating the torque output from the CPG model. We present the details of the two simulation models below.

\subsubsection{Normal Walker}

The CPG was constructed as a set of coupled neural oscillators, each of which is expressed by a set of simultaneous differential equations (Matsuoka 1985). The simultaneous differential equations are shown in Equation (1). Neurons innervating lower limb muscles were mutually coupled so that their oscillations could be entrained to each other; consequently, the skeletal system controlled by the nervous system could display coordinated motion. Figure 3 shows the coupling relations between neurons:

$$
\begin{aligned}
\tau_{n} \dot{u}_{n} & =-u_{n}+\sum_{s} w_{n s} f_{\max }\left(u_{s}\right)_{n}-\beta v_{n}+z_{n}+\text { feed }, \\
\tau_{n}^{\prime} \dot{v}_{n} & =-v_{n}+f_{\max }\left(u_{n}\right), \\
f_{\max }(\xi) & =\max (\xi, 0),
\end{aligned}
$$

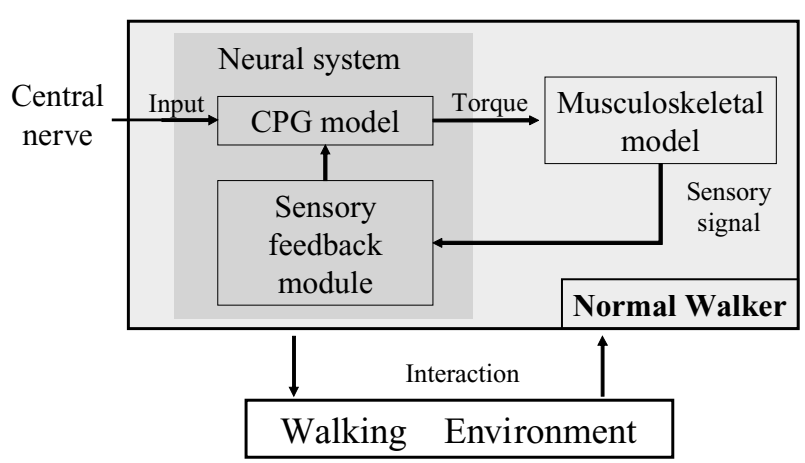

Figure 1. Composition of the Normal Walker and its walking environment.

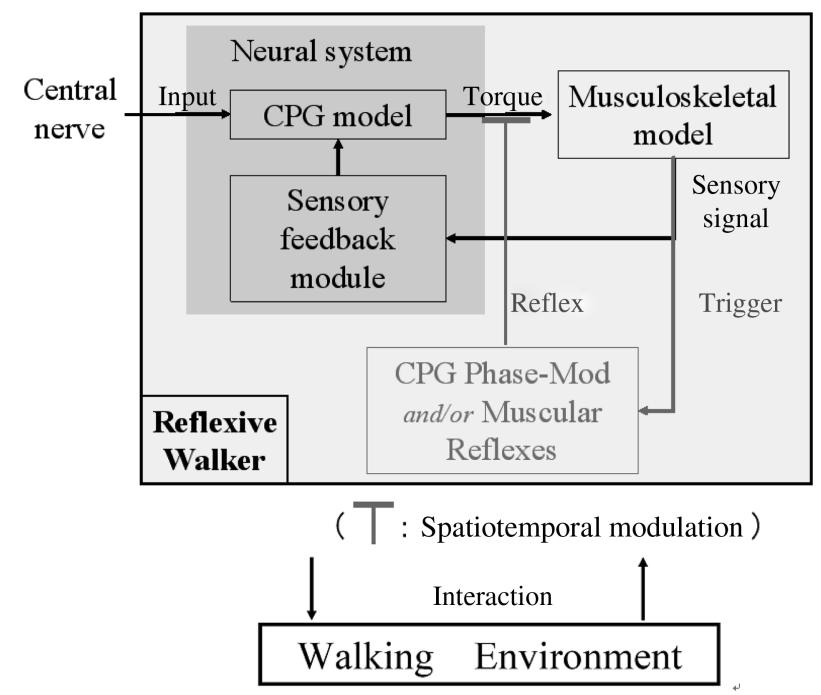

Figure 2. Overview of the Reflexive Walker. 


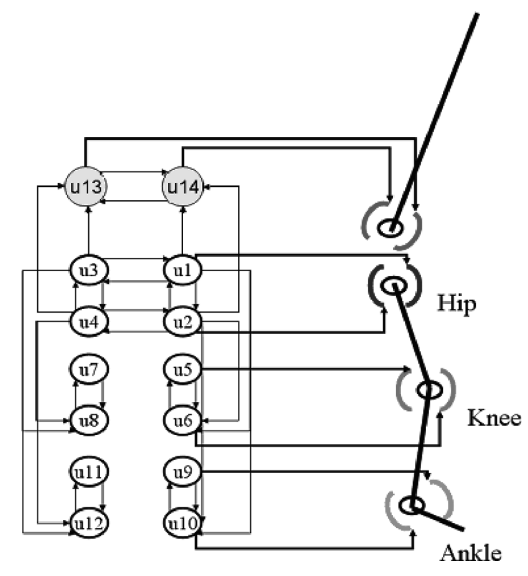

Figure 3. Neuron-neuron and neuron-link connections.

where $u_{n}$ is the inner state of the $n$th neuron; $f_{\max }$ is the output of the $n$th neuron; $v_{n}$ is a variable representing the degree of the adaptation or self-inhibition effect of the $n$th neuron; $z_{n}$ is an external input with a constant rate; $w$ is the connecting weight between coupled neurons; and $\tau$ and $\tau^{\prime}$ are time constants of the inner state and the adaptation effect, respectively. Neuron output $f_{\max }\left(u_{n}\right)$ is treated as the torque generated by the modulated muscle. Torques acting on joints were calculated as the differences of antagonistic muscle pairs.

This neural expression has also been widely used in other walking simulations (Taga 1994; Ogihara and Yamazaki 2001). The feed in Equation (1) can be calculated in Equation 2 (Matsuoka 1985) as follows:

$$
\begin{aligned}
\text { feed }= & A(-X)+B_{\mathrm{R}} h F g_{\mathrm{R}}(-X)+B_{\mathrm{L}} h F g_{\mathrm{L}}(-X) \\
& +C_{\mathrm{R}} h F g_{\mathrm{R}}+C_{\mathrm{L}} h F g_{\mathrm{L}}+D\left(-X_{d}\right),
\end{aligned}
$$

where feed is a vector consisting of 14 elements corresponding to the feedback to 14 neurons (please refer to Figure 3 for the neuron settings) and $X$ is a vector variable expressing the state of the simulated links. $\left(X_{1}, X_{2}\right),\left(X_{3}, X_{4}\right),\left(X_{6}\right.$, $\left.X_{7}\right),\left(X_{9}, X_{10}\right),\left(X_{12}, X_{13}\right),\left(X_{15}, X_{16}\right)$ and $\left(X_{18}, X_{19}\right)$ express the positions of the centre of gravity of the hip joint, left thigh, right thigh, left lower leg, right lower leg, torso and head, respectively. $X_{5}, X_{8}, X_{11}, X_{14}$ and $X_{17}$ express the angle of the left thigh, right thigh, left lower leg, right lower leg and torso, respectively. Correspondingly, $X_{d 5}$, $X_{d 8}, X_{d 11}, X_{d 14}$ and $X_{d 17}$ stand for the angular velocity of the left thigh, right thigh, left lower leg, right lower leg and torso, respectively. $h F g_{R}$ and $h F g_{L}$ are two-value functions, taking a value of 1 during the stance phase and 0 during the swing phase for the right and left sides, respectively. $A, B_{\mathrm{R}}$, $B_{\mathrm{L}}, C_{\mathrm{R}}, C_{\mathrm{L}}$ and $D$ are the coefficient matrices.

Since feed contains pose- and angle-change information of the simulated links, as well as the reaction forces from the ground to the skeletal system, the interaction between the neuromusculoskeletal system and the external world could be realised. Our simulation model also employed this expression.

The weight and size of the body segments were set as follows. The head was set as a point, with a weight of $4 \mathrm{kgf}$. The torso, thigh, lower leg and foot were set as rectangles, whose width $\times$ height pairs are $0.7 \times 0.05,0.5 \times 0.05$, $0.6 \times 0.05$ and $0.25 \times 0.3 \mathrm{~m}$, respectively. Their weights were set as $32,7,4$ and $1 \mathrm{kgf}$, respectively. The relative mass ratios of body segments are approximately in agreement with those of actual humans (Nakamura and Saito 1992).

The model was developed using MATLAB version 7.0 software (The MathWorks, Natick, MA, USA) and Working Model 2D version 7.0 software (MSC Software, Santa Ana, CA, USA). They were coupled by DDE (dynamic data exchange) protocol.

The utility of the simulation model is verified by comparing its joint trajectories during walking with those of a human subject.

\subsubsection{Reflexive Walker}

In order to realise the reflexive responses in the simulator, the following three points, that is, three aspects of the reflex function, should be determined:

(1) Spatial aspect: The muscles that should be activated. In this article, this was decided by the muscular reflexive patterns described in Subsection 2.1 .

(2) Temporal aspect: The onset timing, order of muscle activation and interval of muscle activation. The triggering problem is addressed in a previous study on perturbation occurrence detection (Hagane et al. 2006) and will not be discussed in this article. The order of muscle activation was again decided by the results of the measurement experiment. The interval of muscle activation was difficult to estimate from our human gait experiments, so the value is derived by an optimal search of computer simulation experiments. In this study, we used three different intervals $(0.02,0.05$ and $0.09 \mathrm{~s})$ because the human subject could modulate the strength of muscular reflexes suitably according to the strength of perturbation.

(3) Relation to CPG output: The human walking rhythm is known to show phase shift or 'resetting' in response to external perturbation, and it is believed that such responses correct the perturbed motion and prevent the walker from falling (Yamasaki et al. 2003). Thus, in this study, after muscular reflexive activation, the CPG phase was set to be the same as that before the reflexive activation (CPG phase modulation). Figure 4 


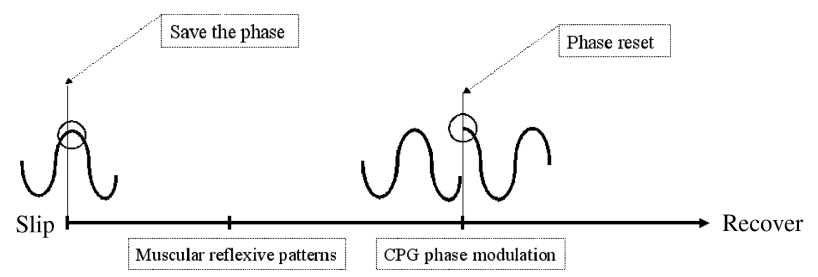

Figure 4. CPG phase modulation.

shows the outline of CPG phase modulation. The effective timing of CPG phase modulation (reset time) was derived from a previous computer simulation experiment.

The slip perturbations to the simulated walkers were implemented by setting the friction coefficient to 0 for a period of time from the moment of heel strike. Different slip intervals would be used as parameters to adjust the levels of the perturbations.

If a walker was able to keep walking for three steps after perturbation, then its balance recovery was judged to be 'successful'; otherwise, balance recovery was judged as 'failed'.

Phase portraits, whose horizontal axis gives the angle and vertical axis gives angular velocity, were used to reveal qualitative data regarding the dynamics of the simulated walkers.

\subsection{Evaluation}

Only the successful/failed evaluation was unable to reflect the difference between the same 'fell' or 'recovered' cases. With regard to quantitative evaluation, we used two stability criteria.

\subsubsection{Energy stability margin}

It is used to evaluate static postures of a walking machine (Messuri and Klein 1985), and defined as 'the potential energy gain by the change of the centre of mass on the occurrence of perturbation' (Equation 3):

$$
S=M\left(h_{\max }-h_{0}\right)
$$

where $M$ is the mass of a walking object; $h_{\max }$ is the height that a COG is changed to on the occurrence of fall; and $h_{0}$ is the current height of the COG.

\subsubsection{Energy difference}

It is proposed for the dynamic evaluation of stability during walking, and defined as 'the difference of the lower limb's rotational energy in swing phase between perturbed and normal walking' (Equation 4):

$$
E D=\frac{1}{2} I \omega_{\text {perturbed }}{ }^{2}-\frac{1}{2} I \omega_{\text {normal }^{2}},
$$

where $I$ is a moment of inertia, and $\omega$ is angular velocity of each lower limb at the time of normal and perturbed walking.

\section{Results}

In this section, we first compare the gaits of perturbed walking of the Normal Walker and the Reflexive Walker to reveal the roles of different functional mechanisms, i.e. phase modulation, muscular reflexive patterns and afferent feedback pathway.

\subsection{Gaits of the Normal Walker and Reflexive Walker}

\subsubsection{Normal Walker}

In cases where no perturbation occurs, the Normal Walker can start from a still state and walk as long as required. The first half of Figure 5 shows stable gait; however, on the occurrence of a slip perturbation (duration: $0.1-0.3 \mathrm{~s}$, illustrated by the red arrow) at the moment of heel strike, the Normal Walker fell down, as shown in the latter half of the stick graph (Figure 5a). The muscular-skeletal model completely lost its balance, falling backwards and failed to carry the rear leg forwards for the next step.

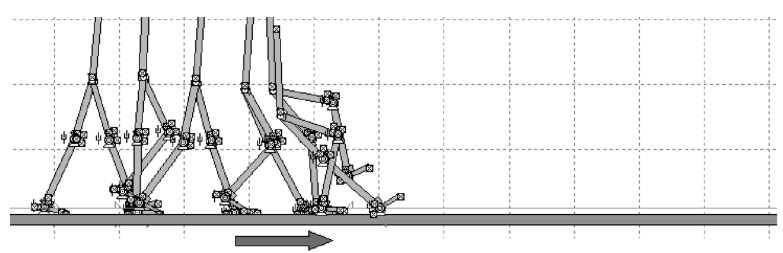

(a)

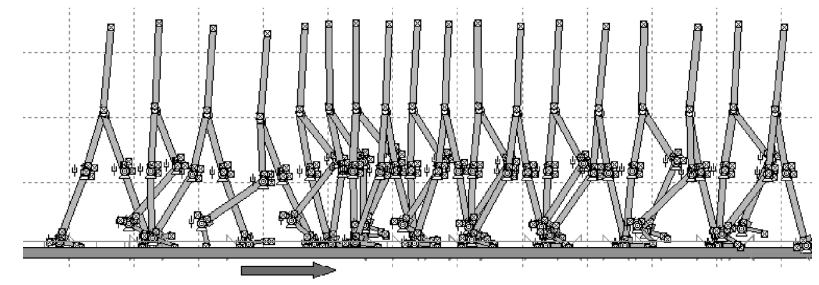

(b)

Figure 5. Stick graphs of the Normal Walker and the Reflexive Walker (arrows denote the slip period): (a) the Normal Walker fell down when a slip perturbation occurs; and (b) the Reflexive Walker recovered from slip perturbation. 


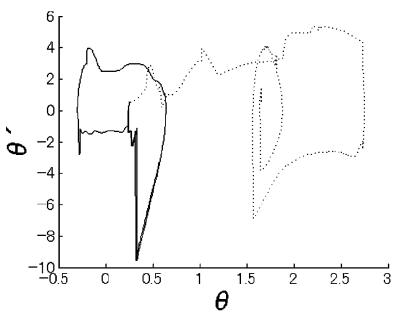

(a)

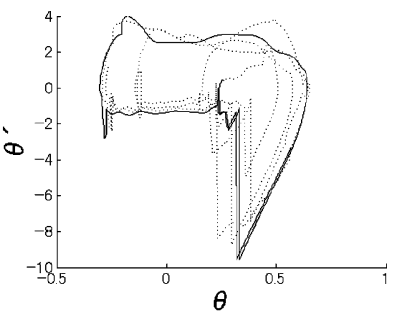

(b)
Figure 6. Phase portraits of hip joint angle: (a) Normal Walker and (b) Reflexive Walker.

However, the tested reflexive muscular patterns, as well as CPG phase modulation, achieved some successful balance recovery cases. Figure $5 \mathrm{~b}$ shows one of the walkers successfully coping with a perturbation, in which the walker initially supported the weight with its rear leg to prevent the body from falling backwards, while simultaneously delaying the timing to move the rear leg forwards for the next step, which is also observed in human gait experiments.

Figure 6 shows the phase portraits of the hip joint angle of the perturbed side. In the phase portrait of the Normal Walker (Figure 6a), the left closed loop stands for a limit cycle denoting walking in the normal condition. After the slip perturbation, the phase trajectory deviated from and could not return to the limit cycle denoting normal walking. However, as shown in Figure 6b, in the case of the Reflexive Walker, after the slip perturbation occurred, the trajectory tended to return to the limit cycle for normal walking. Note that, in Figure 6b, the dotted line shows the trajectory after the slip perturbation occurred.

\subsection{Roles of different regulation mechanisms}

\subsubsection{Phase modulation with muscular reflexive patterns}

It is clear that muscular reflexive patterns and CPG phase modulation could enable the Reflexive Walker to recover from stumbles resulting from slip perturbations. Figure 7 shows the balance recovery by CPG phase modulation and muscular reflexive patterns.

As shown in Figure 7, effective reset time differs by slip duration, and becomes longer as slip duration increases. It is also observed that a longer muscular reflex (activation period $=0.09 \mathrm{~s}$ ) is effective for larger perturbations, and a shorter muscular reflex (activation period $0.02 \mathrm{~s}$ ) is effective for smaller perturbations.

For example, the case in the circle means 'The walker recovered by CPG phase modulation (reset time $0.22 \mathrm{~s}$ ) and muscular reflexive patterns (activation period $0.05 \mathrm{~s}$ ) against 0.25 -s slip perturbation'.

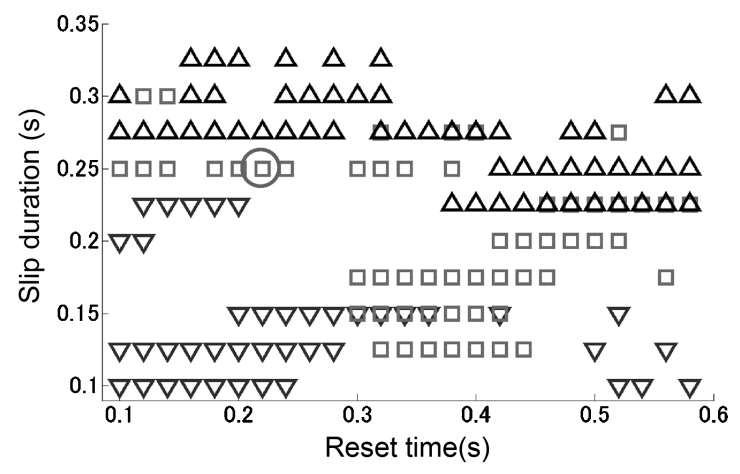

Figure 7. Balance recovery by CPG phase modulation and muscular reflexive patterns when slip perturbation duration changes. The 'reset time' is a parameter of CPG phase modulation, meaning the timing at which CPG restarts from a phase memorised at the occurrence of a slip perturbation, counting from the time of perturbation occurrence. A blank (no mark) stands for a complete loss of its balance at the corresponding reset time and slip duration. Different marks $(\nabla, \square$ and $\Delta)$ indicate the following recovery cases: Blank Fell down though with muscular reflexive patterns $\nabla$ Recovery with muscular reflexive patterns (activation period $=$ $0.02 \mathrm{~s}$ ). $\square$ Recovery with muscular reflexive patterns (activation period $=0.05 \mathrm{~s}) . \Delta$ Recovery with muscular reflexive patterns (activation period $=0.09 \mathrm{~s}$ ).

\subsubsection{Phase modulation without muscular reflexive patterns}

Another possibility requiring inspection is whether CPG phase modulation alone could result in types of balance recovery. In order to clarify this point, sets of experiments were conducted. Figure 8 shows the results of the comparison between a CPG phase modulation alone case (' + ' in Figure 8) and CPG phase modulation and muscular reflex case ('o' in Figure 8). As shown in the figure, even CPG phase modulation alone could lead to several kinds of perturbation resistance. However, in the case of slip durations over $0.3 \mathrm{~s}$, no reset times led to a successful balance

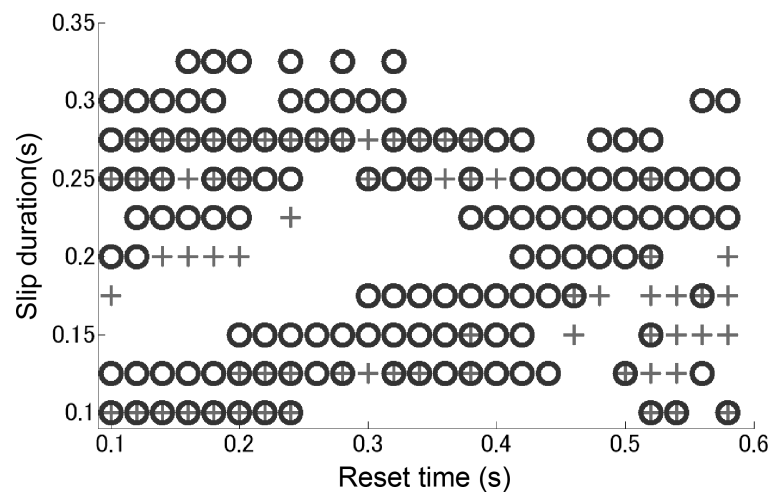

Figure 8. Balance recovery in the $\mathrm{CPG}$ phase modulation alone case $(+)$ and the CPG phase modulation with the muscular reflexive pattern case $(0)$ when duration of slip perturbation changes. 
Table 2. Balance recovery by muscular reflexive patterns when duration of slip perturbation changes.

\begin{tabular}{ccccccccccc}
\hline Slip duration (s) & 0.1 & 0.125 & 0.15 & 0.175 & 0.2 & 0.225 & 0.25 & 0.275 & 0.3 & 0.325 \\
\hline 0.02 -s Activation & $\mathrm{o}$ & $\mathrm{o}$ & $\mathrm{o}$ & $\times$ & $\times$ & $\times$ & $\times$ & $\times$ & $\times$ & $\times$ \\
0.05 -s Activation & $\times$ & $\times$ & $\times$ & $\times$ & $\times$ & $\times$ & 0 & $\times$ & $\times$ & $\times$ \\
0.09 -s Activation & $\times$ & $\times$ & $\times$ & $\times$ & $\times$ & $\times$ & $\mathrm{o}$ & $\times$ & $\mathrm{o}$ & $\times$ \\
\hline
\end{tabular}

Note: ' $o$ ' indicates recovered; $\times$, fell down.

recovery. It is clear that the ability to recover takes advantage of the activation of the muscular reflexive patterns.

\subsubsection{Muscular reflexive patterns without phase modulation}

Similarly, the muscular-reflex-alone case should also be investigated. Table 2 shows the results of balance recovery by muscular reflexive patterns alone. The three muscular reflexive pattern activation periods $(0.02,0.05$ and $0.09 \mathrm{~s})$ were applied. As shown in Table 2, even muscular reflex alone can lead to several types of perturbation resistance, although only to a very limited extent. Moreover, Figure

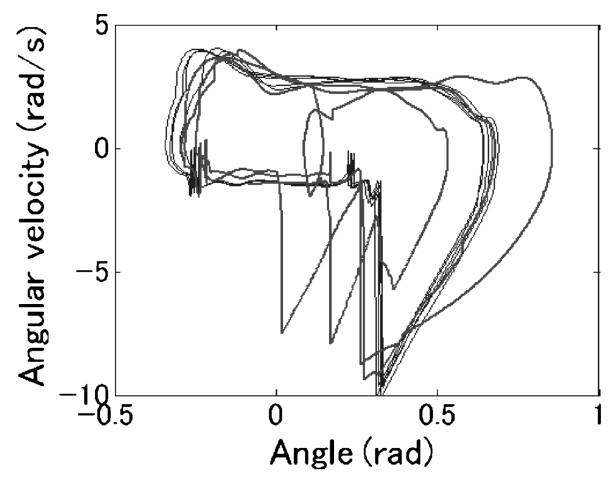

(a) Muscular-reflex-alone case

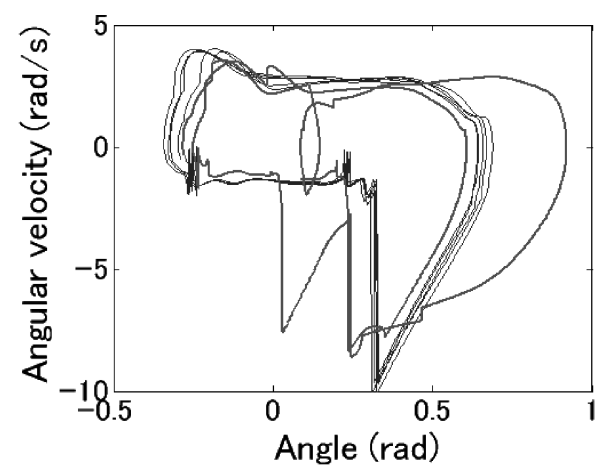

(b) Reflexive Walker (Muscular reflex + Phase modulation)

Figure 9. Phase portraits of the hip joint angle. The lines represent normal/perturbed walks. It was observed that (a) the muscular-reflex-alone case returned to the attractor in $2.9 \mathrm{~s}$, while (b) the Reflexive Walker returned to the attractor in $1.9 \mathrm{~s}$.
$9 \mathrm{a}$ and $9 \mathrm{~b}$ show the hip joint angle phase portraits of the muscular-reflex-alone case and the muscular reflex with phase modulation case, respectively. Note that the slip condition and the muscular-reflexive patterns activation period are same for both tests, and only the hip angle values of the perturbed side are plotted. It was observed that the Reflexive Walker could return back to the normal walking attractor within $1.9 \mathrm{~s}$, while muscular reflex alone could lead the walker back to the normal walking attractor within $2.9 \mathrm{~s}$.

\subsubsection{Evaluation}

In order to examine the role of reflexive mechanisms, muscular reflexive patterns and CPG phase modulation, we evaluated the perturbed walk of each walker (Normal Walker, Normal Walker with phase modulation, Normal Walker with muscular reflex and Reflexive Walker) by the energy stability margin and energy difference (see section 2.4). Five seconds of the energy stability margin of each walker in the slip-perturbed condition (0.2-s slip) are plotted in Figure 10. Here, slip perturbation occurs at $1 \mathrm{~s}$ in Figure 10. In order to make a clear comparison, we selected an experimental condition (slip duration $=0.25 \mathrm{~s}$ ) that could lead to successful balance recovery for all four walkers. Note that, for the Normal Walker, this is the rare successful balance recovery case. Additionally, Figure 11 shows the integration of the energy stability margin of each walker after perturbation occurrence. Furthermore, $5 \mathrm{~s}$ of energy difference of each walker in the same slip perturbed condition is plotted in Figure 12. Figure 13 shows the integration of the energy difference of each walker after perturbation occurrence. Arrows in the figures denote points for further discussion. As shown in Figures 10 and 12, the Reflexive Walker has better balance recovery ability than the Normal Walker. It is also observed that both CPG phase modulation and muscular reflexive patterns could achieve more effective balance recovery than the Normal Walker (excepting the energy stability margin value of the phasemodulation-alone case, which will be discussed later), and the Reflexive Walker (utilising both mechanisms) could achieve most effective balance recovery (Figures 11 and 13). This suggests that CPG phase modulation and muscular reflexive patterns display a cumulative effect for balance recovery. 


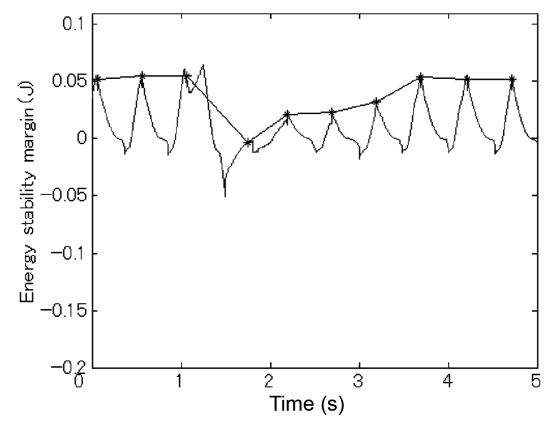

(a)

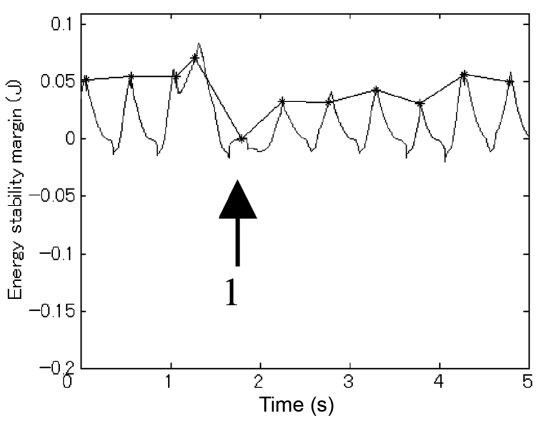

(c)

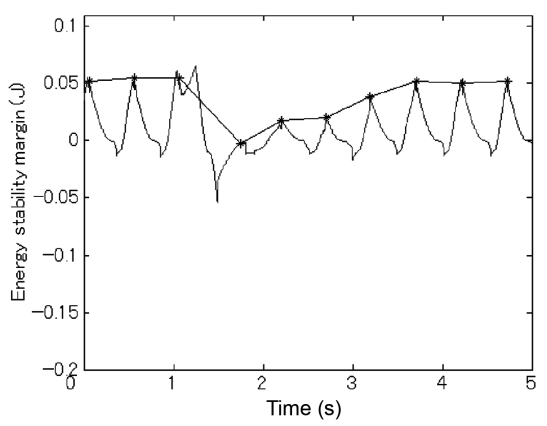

(b)

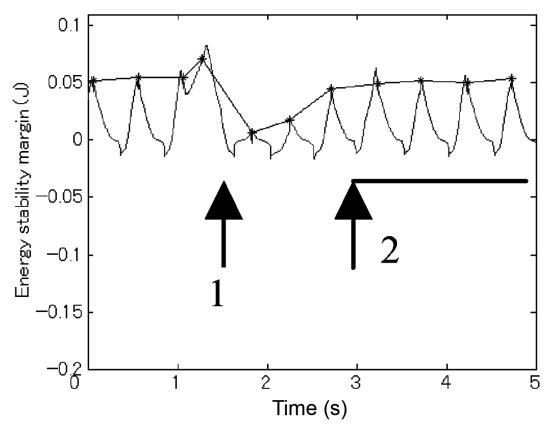

(d)

Figure 10. Energy stability margin: (a) Normal Walker, (b) phase modulation alone, (c) muscular reflex alone and (d) Reflexive Walker. Arrow 1 stands for the immediate improvement of balance after perturbation. Arrow 2 shows the beginning of recovered gait.

\subsubsection{Feedback during normal walking and perturbed walking}

Since the feedback in Equation (1) stands for the information from the afferent pathway, it should also be able to contribute to the balance maintenance. In Figure 14b, on the occurrence of a 0.3 -s slip perturbation, the feedback values of three different walkers, i.e. the Normal Walker, the Normal Walker with CPG phase modulation and the Reflex Walker, are plotted with a thin line, dotted line and

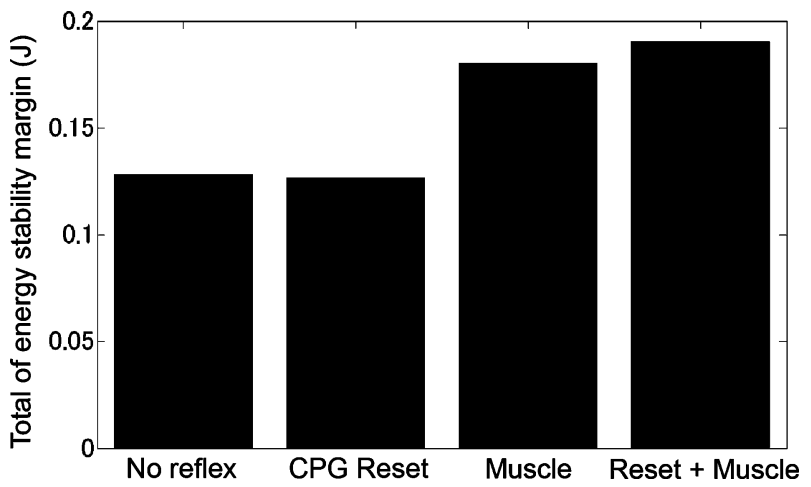

Figure 11. Integration of Energy stability margin (from left, Normal Walker, Normal Walker with phase modulation, Normal Walker with muscular reflex, Reflexive Walker). broken line, respectively. Five seconds of feedback output data of the Normal Walker in normal condition are plotted in Figure 14a. Circle (o) and square ( $\square$ ) denote the beginning of the slip perturbation and the moment of phase restoration, respectively. During the period denoted by circle and square, there is a peak in the broken line (the Reflex Walker), which is the result of the muscular reflexive patterns. After the moment denoted by square, the dotted line (CPG phase modulation alone) exceeds the thin line (Normal Walker), which is the result of phase restoration. Although the Normal Walker finally failed to recover from the perturbation, indicating that feedback alone is not enough to resist the perturbation; nevertheless, as in the case of the Reflex Walker, it is the feedback that drove the walker back to the normal stable limit cycle after the prompt activation of the muscular reflexive patterns and CPG phase modulation. That is, the feedback is not reactive to the perturbation; however, it undoubtedly contributes to the long-term balance recovery process.

\section{Discussion}

The present study demonstrated that perturbation resistance could be improved by the reflexive responses that result from a phase-modulation mechanism and the activation of muscular reflexive patterns. The results are in partial agreement with the outcome from the electroneurograms data 


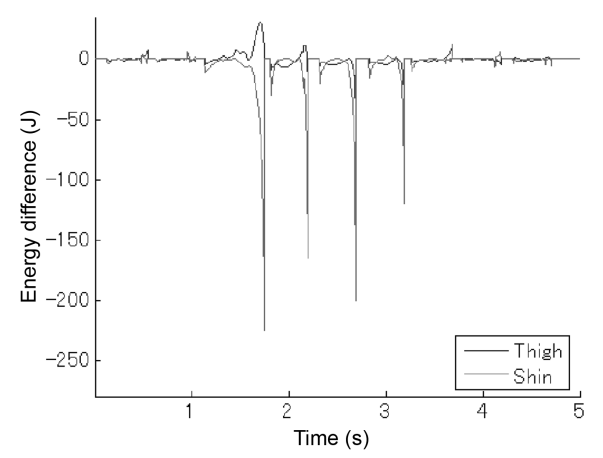

(a)

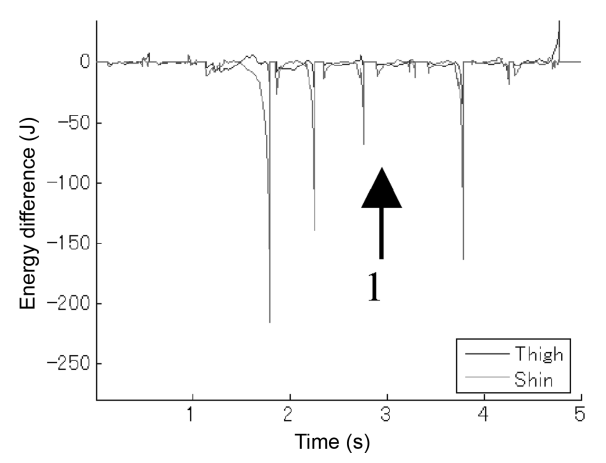

(c)

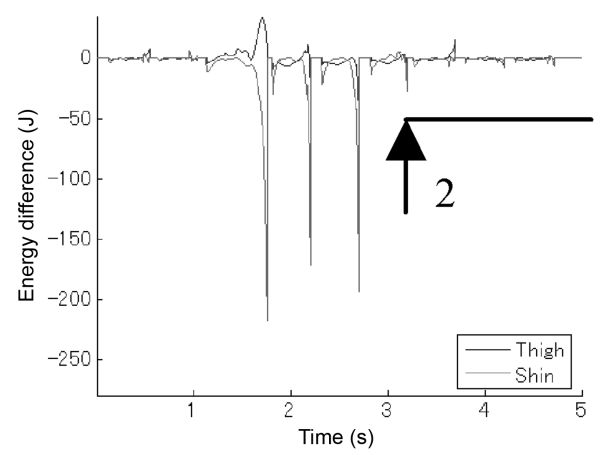

(b)

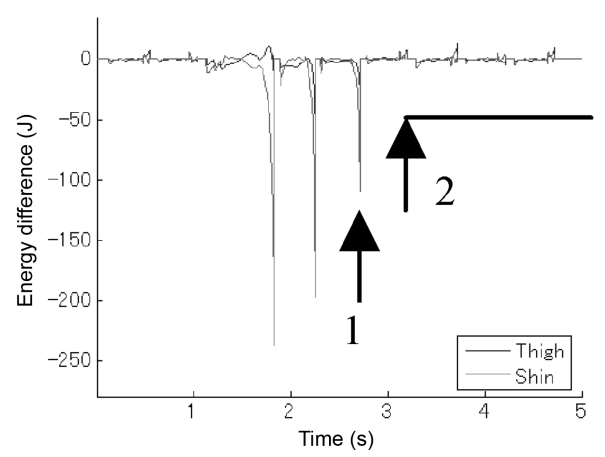

(d)

Figure 12. Energy difference: (a) Normal Walker, (b) phase modulation alone, (c) muscular reflex alone and (d) Reflexive walker. Arrow 1 indicates the immediate improvement of balance after perturbation, by a much smaller energy difference, compared with the fourth drop in the Normal Walker case. Arrow 2 denotes the beginning of the recovered gait, where the walker possesses the same rotational energy as that of normal walking.

from an animal experiment (Schomburg et al. 1998), which proposes that the flexor reflex affects gait phase resetting. Our study further elucidates the experimental results on a neural control level.

We also confirmed that, although the feedback pathway could contribute to the long-term balance recovery process,

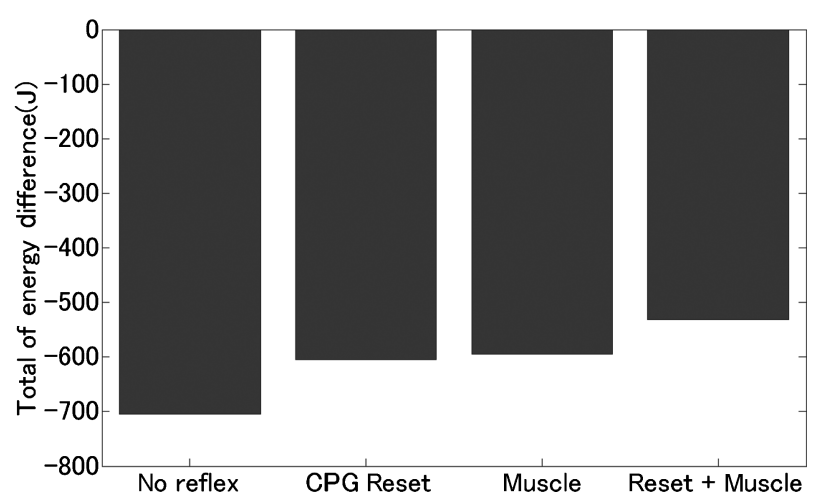

Figure 13. Integration of energy difference (from left, Normal Walker, Normal Walker with phase modulation, Normal Walker with muscular reflex, Reflexive Walker). it is not reactive to perturbations. However, from a control theory viewpoint, an adaptive gain scheduling might possibly result in a similar effect by reflexive responses. This issue requires further studies in both physiology and control theory.

Moreover, successful/failed evaluation of balance recovery may neglect some important aspects and factors. Thus, we conducted a qualitative evaluation of walking and dynamical responses to perturbation using static and kinetic methods (energy stability margin and energy difference), and examined the role of CPG phase modulation and the muscular reflex.

Our results suggest that the muscular reflex is effective for balance recovery immediately after perturbations, particularly large perturbations (Figures 8, 10 and 12). Conversely, CPG phase modulation is effective for modulating the relationship between internal CPG and the body mechanisms enabling a fast recovery to a stable walking cycle (Figures 9, 10 and 12).

Moreover, the two evaluation criteria, energy stability margin and energy difference, both have merits and demerits. Energy difference could reflect the balance recovery improvement by the phase modulation mechanism, while 


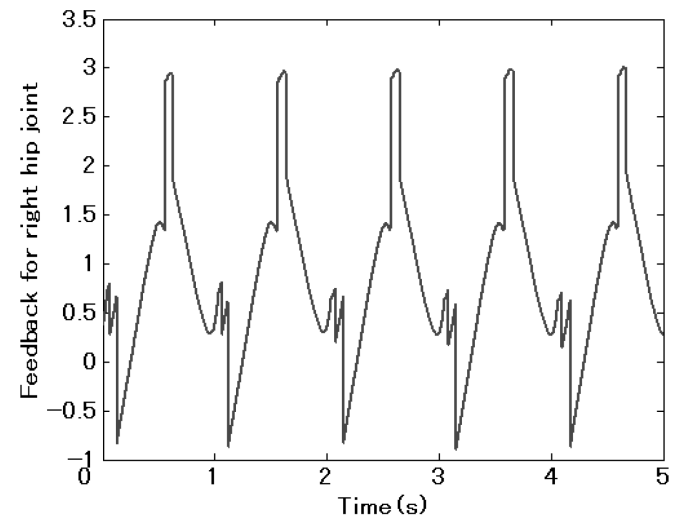

(a) Normal walking

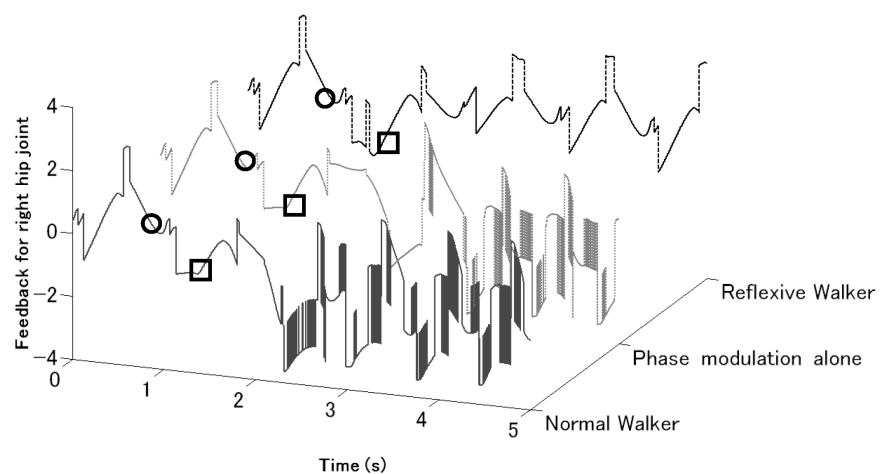

(b) Perturbed walking

Normal Walker

Normal Walker with CPG-phase-modulation-alone Reflexive Walker (CPG-phase-modulation + muscular reflex)

Figure 14. Transition of feedback for right hip joint. Horizontal axis is the elapsed time (s); vertical axis is the amplitude of feedback. Slip perturbation starts from $1.09 \mathrm{~s}$, duration $0.3 \mathrm{~s}$. Circle (o) stands for the beginning of the slip perturbation. Square ( $\square$ ) shows the moment of phase restoration. Only the Reflexive Walker recovered from the perturbation and returned to stable gait after $2.0 \mathrm{~s}$.

energy stability margin could not (Figures $10 \mathrm{~b}, 12 \mathrm{~b})$. This is because the former takes motion (i.e. the rotation of the lower limbs) into consideration, while the latter only reflects differences in posture. In contrast, as shown in Figure 12, the energy difference values of all walkers remarkably decreased immediately after the perturbation. This is because the behaviour of the models immediately after a perturbation is deviated from that of normal walking, regardless of whether certain reflexive mechanisms are involved. In such cases, the difference in posture may reflect the essence of balance recovery function. Thus, immediately after a perturbation, energy stability margin may serve as a better evaluation criterion.

Nevertheless, these results showed that the playback of muscular reflexive patterns and the phase reset of gait are effective for balance recovery from slip perturbation, thus revealing the practical possibility of realising artificial reflexes for paralysed individuals.

However, in order to reach our ultimate goal, two additional issues, stated below, should be clarified.

(1) Perturbations could be detected immediately after their occurrence so that the muscular patterns could be 'played back' by robotic devices or FES to assist in the balance recovery of walking-impaired individuals. In a gait experiment described previously (Hagane et al. 2006), three triaxis accelerometers + gyro sensors (GYROCUBE/3A, O-Navi San Diego, CA, USA) were fitted on the head, waist and ankle. Triaxial accelerometers have previously been used to classify (Mathie et al. 2004) and detect (Mathie et al. 2003) daily physical activities. In our previous related study, we confirmed that, using the aforementioned portable accelerometer and gyro- scopes, slip perturbation could be detected within $100 \mathrm{~ms}$ after occurrence (Hagane et al. 2006). In the future, we will investigate the effects of different playback delays of muscular reflexive patterns on balance recovery. Moreover, since the balance recovery behaviour might have a strong individual difference, we should take the aspect into consideration. Also, according to (Zehr and Stein 1999), reflexive responses are phase-dependent, whereas in this study, we only investigated the slip occurrence at heel-strike moment, the most dangerous slippery case. This will be another issue for further investigation.

As our future work, the balance recovery behaviour will be explored as the result of an interaction between neural, skeletal and muscular mechanisms, and environment. In that case, the delay might emerge from the exploration.

(2) The muscular reflexive patterns for normal subjects may be sufficiently similar so that a representative muscular pattern could be established and employed. However, for walking-impaired individuals, the muscular patterns that are effective in attaining balance recovery may be different, depending on the cause, degree and stage of their impairment. Individual-dependent muscular reflexive patterns will be the next focus of our research.

\section{Conclusion}

In the present study, we developed two simulation models and, through computer simulation, revealed that (1) the simulation model could present conformable behaviour to human normal walking; (2) in the case of occurrence of 
a slip perturbation, the rapid responding pathway could improve perturbation resistance and maintain balance for walking; and (3) using the simulation models, the roles of the muscular reflexive patterns, CPG phase modulation and afferent feedback were clarified.

That is, feedback pathway, phase modulation and reflexive muscular patterns play different but significant roles in balance recovery. Several hypotheses on underlying neuromechanism were also investigated. These results demonstrated the practical possibility of realising artificial reflexes for paralysed individuals.

\section{Acknowledgements}

This work was supported in part by a Grant-in-Aid for Scientific Research (B), 2007, 19300199, from the Ministry of Education, Culture, Sports, Science and Technology of Japan, and by the Kurata Memorial Hitachi Science and Technology Foundation.

\section{References}

Bajd T, Stefancic M, Zmatjacic Z, Kraij A, Savrin R, Benko H, Karcnik T, Obereza P. 1997. Improvement in step clearance via calf muscle stimulation. Med Biol Eng Comp. 35:113-116.

Berger W, Dietz V, Quintern J. 1984. Corrective reactions to stumbling in man: neuronal co-ordination of bilateral leg muscle activity during gait. J Physiol. 357:109-125.

Bisdorff R, Bronstein AM, Wolsley C, Gresty MA, Davies A, Young A. 1999. EMG responses to free fall in elderly subjects and akinetic rigid patients. J Neurol Neurosurg Psychiatry. 66:447-455.

Cathers I, O'Dwyer N, Neilson P. 2004. Variation of magnitude and timing of wrist flexor stretch reflex across the full range of voluntary activation. Exp Brain Res. 157:324-335.

Dietz V, Quintern J, Sillem M. 1987. Stumbling reactions in man: significance of proprioceptive and pre-programmed mechanisms. J Physiol. 386:149-163.

Hagane Y, Yu W, Katane T, Sekine M, Tamura T, Saitou O. 2006. Detecting perturbation occurrence during walking. 11th ICPE, International Conference on Precision Engineering. Tokyo, Japan, August 2006.

Kawamoto H., Kanbe S, Sankai Y. 2003. Power assistmethod for HAL-3 estimating operator's intension based on motioninformation. Proceedings of 12th IEEE Workshop on Robot and HumanI nteractive Communication (ROMAN 2003). CA, USA.
Mathie MJ, Celler BG, Lovell NH, Coster ACF. 2004. Classification of basic daily movements using a triaxial accelerometer. Med Bio Eng Comp. 42:679-687.

Mathie MJ, Coster ACF, Lovell NH, Celler BG. 2003. Detection of daily physical activities using a triaxial accelerometer. Med Bio Eng Comp. 41:296-301.

Matsuoka K. 1985. Susteined oscillation generated by mutually inhibiting neurons with adaptation. Biol Cybern. 52: 367-376.

Messuri DA, Klein CA. 1985. Automatic body regulation for maintaing stability of a legged vehicle during rough-terrian locomotion. IEEE J Robot Auomat. RA-1(3) 132-141, September.

Nakamura R, Saito H. 1992. Basic kinesiology. 4th ed. Ishiyaku: Tokyo.

Ogihara N, Yamazaki N. 2001. Generation of human bipedal locomotion by a bio-mimetic neuromuscloskeletal model. Biol Cybern. 84:1-11.

Prochazka A, Yakovenko S. 2001. Locomotor control: from spring-like reactions of muscles to neural prediction. In: Nelson RJ, editor. The somatosensory system: deciphering the brain's own body image. Boca Raton: CRC Press. p. $141-181$.

Quintern J. 2000. Stimulation of flexor reflex afferents: a pathway to the spinal pattern generator for human locomotion? 6th Internet World Congress for Biomedical Sciences, Presentation $\# 60$, online conference.

Schomburg ED, Petersen N, Barajon I, Hultborn H. 1998. Flexor reflex afferents reset the step cycle during fictive locomotion in the cat. Exp Brain Res. 122:339-350.

Taga G. 1994. Emergence of bipedal locomotion through entrainment among the neuromusculoskeletal system and the environment. Physica D. 75:190-208.

Tong YT, Granat MH. 1998. Reliability of neural-network functional electrical stimulation gait-control system. Med Biol Eng Comput. 37:633-638.

Yamasaki T, Nomura T, Sato S. 2003. Possible function roles of phase resetting during walking. Biol Cybern. 88:468496.

Yu W, Ikemoto Y. 2007. An artificial reflex improves the perturbation-resistance of a human walking simulator. Med Biol Eng Comput. 45:1095-1104. Special Issue of World Congress on Medical Physics and Biomedical Engineering 2006, Springer. doi 10.1007/s11517-007-0255-1.

Yu W, Yamaguchi H, Yokoi H, Maruishi M, Mano Y, Kakazu Y. 2002. EMG automatic switch for FES control for hemiplegics using artificial neural network. Robot Auton Syst. 40:213224.

Zehr EP, Stein RB. 1999. What functions do reflexes serve during human locomotion? Prog Neurobiol. 58:185-205. 

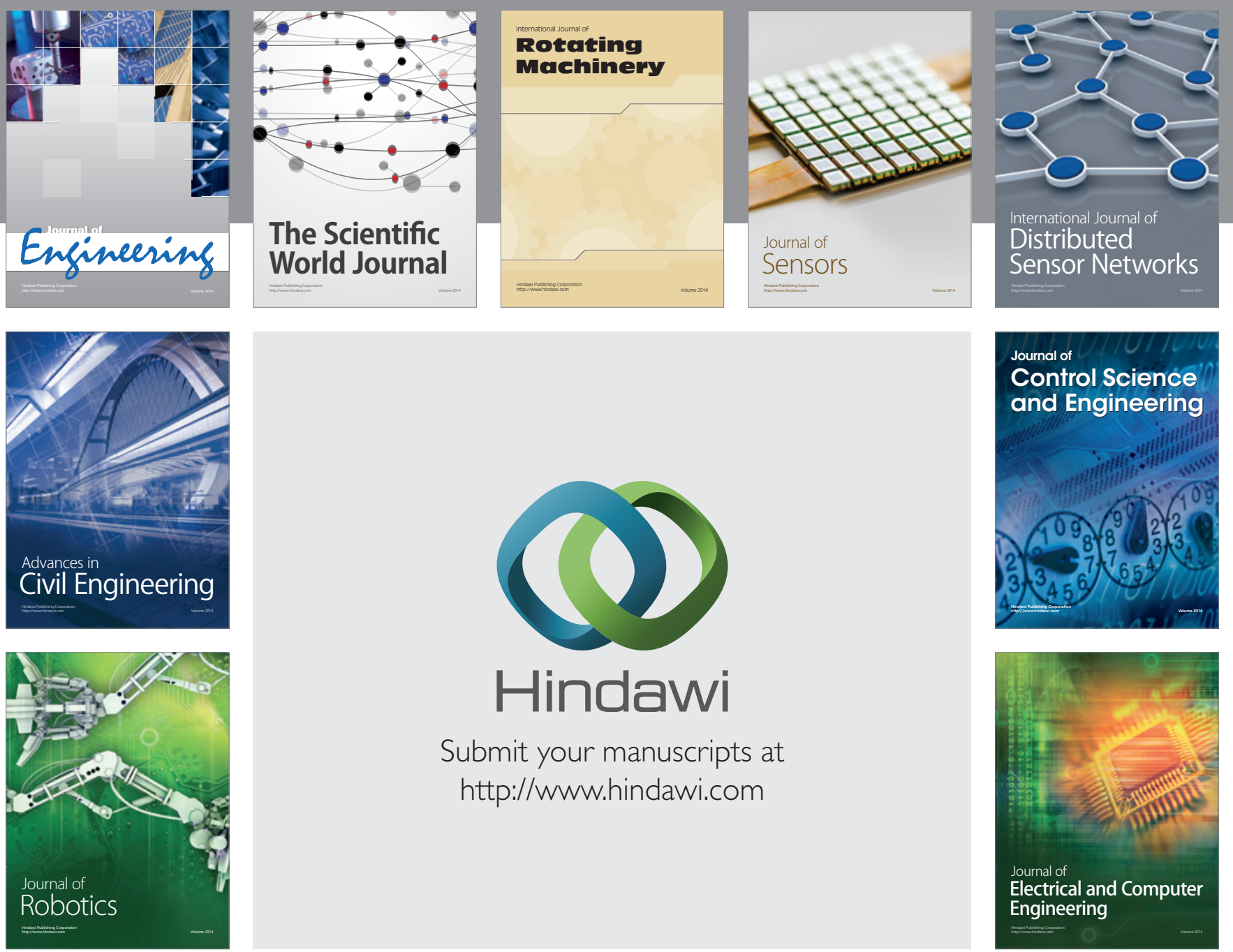

Submit your manuscripts at

http://www.hindawi.com
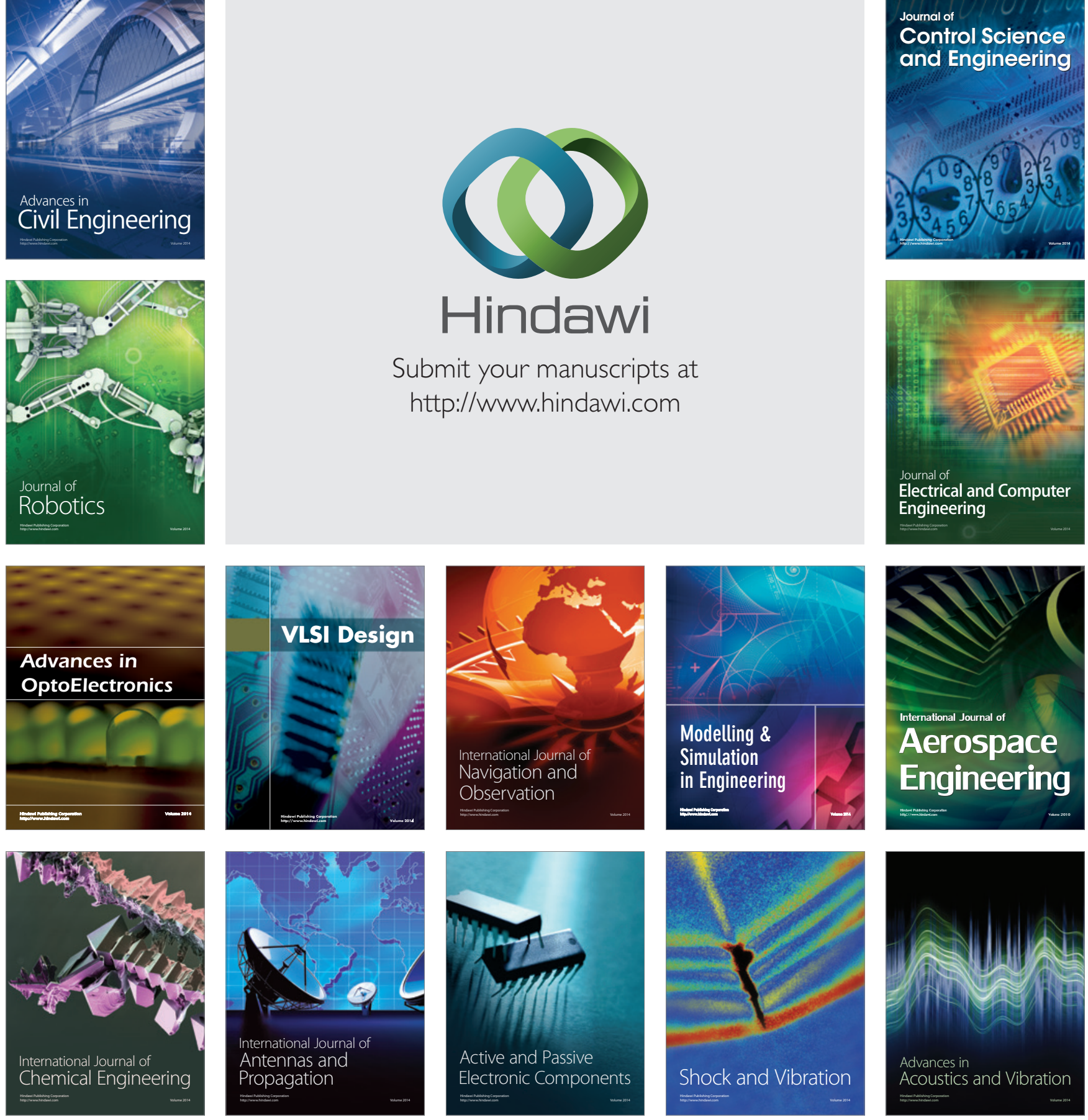